\title{
Erratum
}

\section{On the $K$-theory of the classifying space of a discrete group}

\section{Alejandro Adem}

Mathematics Department, University of Wisconsin, Madison, WI 53706, USA

Math. Ann. 292, 319-327 (1992)

A typographical error occurred in the statements and proofs of the main results (Theorem 3.1 and Theorem 4.2) on pages 320,324, and 326. They should read as follows:

On page 320:

\section{Theorem 3.1.}

$$
K_{G}^{*}\left(X / \Gamma^{\prime}\right) \otimes \mathbb{C} \cong K^{*}\left(B \Gamma^{\prime}\right)^{G} \otimes \mathbb{C} \oplus \bigoplus_{\gamma \in \zeta-\{1\}} K^{*}\left(B\left(C(\gamma) \cap \Gamma^{\prime}\right)\right)^{H_{\gamma}} \otimes \mathbb{C}
$$

where $\mathcal{Y}$ is the set of conjugacy classes of elements of finite order in $\Gamma$, and $H_{\gamma}=$ $C(\gamma) / C(\gamma) \cap \Gamma^{\prime}$, a finite group.

On page 324:

Theorem 3.1.

$$
K_{G}^{*}\left(X / \Gamma^{\prime}\right) \otimes \mathbb{C} \cong \bigoplus_{(\gamma)} K^{*}\left(B\left(C(\gamma) \cap \Gamma^{\prime}\right)\right)^{H_{\gamma}} \otimes \mathbb{C}
$$

where $\gamma$ ranges over conjugacy classes of elements of finite order in $\Gamma$, and $H_{\gamma}=$ $C(\gamma) / C(\gamma) \cap \Gamma^{\prime}$, a finite group.

Proof. Simply combine (2.2) with (1.4) and identify

$$
\begin{aligned}
K^{*}\left(X^{\langle\gamma\rangle} / C(\gamma)\right) \otimes \mathbb{C} & =K^{*}\left(\left[X^{(\gamma\rangle} / C(\gamma) \cap \Gamma^{\prime}\right] / H_{\gamma}\right) \otimes \mathbb{C} \\
& =K^{*}\left(B\left(C(\gamma) \cap \Gamma^{\prime}\right)\right)^{H_{\gamma}} \otimes \mathbb{C} .
\end{aligned}
$$


On page 326 :

Theorem 4.2.

(i)

$$
K_{p}^{*}(B \Gamma) \otimes \mathbb{C}_{p} \cong \bigoplus_{\substack{(\gamma) \\ \gamma \in \Gamma(p)}} K_{p}^{*}\left(B \Gamma^{\prime} \cap C(\gamma)\right)^{H_{\gamma}} \otimes \mathbb{C}_{p}
$$

(ii)

$$
\chi\left(K_{p}^{*}(B \Gamma) \otimes \mathbb{C}_{p}\right)=\sum_{\substack{(\gamma) \\ \gamma \in \Gamma(p)}} \tilde{\chi}(C(\gamma))
$$

Example 4.3. Now let $\Gamma=\mathrm{SL}_{3}(\mathbb{Z}), \Gamma^{\prime}=\Gamma(3), G=\mathrm{SL}_{3}\left(\mathbb{F}_{3}\right)$. Note $|G|=2^{4} \cdot 3^{3} \cdot 13$ but that $\Gamma$ has no 13-torsion. Hence we deduce

$$
K_{p}^{*}(B \Gamma) \otimes \mathbb{C}_{p} \cong \bigoplus_{\substack{(\gamma) \\ \gamma \in \Gamma(p)}} K^{*}(B C(\gamma) \cap \Gamma(3))^{H_{\gamma}} \otimes \mathbb{C}_{p} \quad \text { for } \quad p=2,3
$$

The statement at the end of the Example 4.3 is inaccurate. It should be modified to say ... "the centralizers of elements of order 2 or 4 in $\mathrm{SL}_{3}(\mathbb{Z})$ are rationally acyclic"... . The conclusions remain valid.

Finally, it has been brought to the author's attention that the result attributed to Atiyah and Segal on p. 320 of [A-S2] was proved by N.J. Kuhn in: Character rings in algebraic topology. Advances in Homotopy Theory (London Math. Soc. Lect. Note Ser., vol. 139), Salamon, Steer and Sutherland (eds.). Cambridge: Cambridge University Press 1989. 